\title{
Sono e treinamento em atletas de elite do Estado de Santa Catarina, Brasil
}

CDD. 20.ed. 613.79

796.073

http://dx.doi.org/10.1590/1807-55092015000200207
Fernanda Tolentino de Souza BLEYER* Rubian Diego ANDRADE* Clarissa Stefani TEIXEIRA ${ }^{* *}$ Érico Pereira Gomes FELDEN*
*Centro de Ciências da Saúde e Esporte, Universidade do Estado de Santa Catarina.

${ }^{* *}$ Centro Tecnológico, Universidade Federal de Santa Catarina.

\section{Resumo}

Objetivou-se investigar algumas questões de sono, saúde e treinamento de atletas de elite do Estado de Santa Catarina - SC. Fizeram parte da amostra 452 atletas de modalidades coletivas e individuais que responderam a um questionário com questões relacionadas ao sono (duração e qualidade do sono, pesadelos e piora do sono antes da competição) e sobre sua percepção de saúde. Além disso, foi investigado se os atletas receberam, durante seus treinamentos, recomendações a respeito do seu sono. 48,5\% dos atletas apresentaram baixa duração do sono $(<8 h)$. Aqueles que receberam instruções sobre sono no treinamento apresentaram maior duração do sono $(p<0,001)$ e melhor qualidade de sono $(p=0,045)$. Atletas que não tiveram instruções sobre o sono apresentaram 1,35 (IC95\%1,02-1,78) vez mais prevalência de baixa duração do sono. Além disso, a maioria dos atletas relatou que seu sono piora na véspera de competições e jogos importantes. Uma boa qualidade do sono esteve associada com percepção mais positiva de saúde e aqueles atletas que receberam instruções sobre o sono durante seus treinamentos apresentaram maior duração e melhor qualidade do sono. Recomenda-se assim, que as instruções sobre sono passem a fazer parte das rotinas de treinamentos de atletas brasileiros, bem como se investigue quais informações e estratégias são mais efetivas para a melhora do sono dos atletas.

Palavras-chave: Privação de sono; Atletas; Saúde.

\section{Introdução}

O ciclo vigília/sono é um ritmo biológico e está ligado a inúmeros eventos fundamentais para a sobrevivência, bem como para um bom desempenho motor e esportivo ${ }^{1-2}$. Neste contexto, no caso dos atletas, que utilizam seu corpo como ferramenta de trabalho, faz-se necessário, além do planejamento dos treinamentos específicos das modalidades, uma organização dos períodos de recuperação incluindo rotina de noites bem dormidas ${ }^{3-5}$.

O sono é apontado como importante fator na manutençáo do grau de alerta e rendimento geral do atleta visando o aproveitamento máximo de seu potencial ${ }^{6-9}$. Além disso, a competição sugere uma série de mudanças no comportamento do atleta que podem ser positivas ou negativas.

Relatos em estudos com atletas adultos e adolescentes mostraram que, durante a competição, um dos principais causadores do estresse é a falta do repouso e problemas com acomodaçóes ${ }^{10}$. Assim, conhecer melhor o padráo do sono de quem pratica atividade física, seja com foco na saúde ou competição, bem como o efeito das atividades esportivas neste padrão, pode contribuir para prescriçōes de treinamento mais adequados. Mesmo que não se tenha pontos de corte identificando a quantidade necessária de sono para o bom rendimento do atleta, até mesmo porque a quantidade ideal de sono é individual, sabe-se que a privação do sono diminui o rendimento e que o sono estendido para além do habitual pode melhorar a performance motora do atleta $^{2,11-15}$. MAH et al. ${ }^{2}$ sugere em seus estudos que o sono estendido pode contribuir para um melhor desempenho atlético, menor tempo de reação, melhor humor e vigor físico e diminuição da fadiga. 
Desta forma, a literatura aponta para a relevância do sono no desempenho esportivo e para o sucesso das competiçóes atléticas. No entanto, é possível identificar importantes lacunas de conhecimento científico a respeito do sono dos atletas. Destaca-se a ausência de estudos que investiguem os ritmos biológicos em atletas brasileiros, bem como se os mesmos

\section{Método}

Participaram da amostra 452 atletas (276 feminino e 176 masculino). A população deste estudo foi formada pelos atletas de elite, de diferentes modalidades coletivas (voleibol, futsal, futebol de campo, handebol, basquete) e individuais (atletismo, ginástica rítmica e artística, tiro), inscritos na maior competição poliesportiva do Estado de Santa Catarina - SC, Brasil, que ocorre anualmente. Tais equipes são representativas de diferentes regióes do estado e foram os mais bem classificado em suas regiôes após três etapas competitivas eliminatórias (municipal, micro-regional e regional). Para o cálculo amostral considerou-se o número de atletas da última competição (4720 atletas) e foi utilizada a equação apresentada por RoDRIGUEs ${ }^{16}$ considerando um erro amostral de 5\%, indicando um número mínimo de 356 atletas.

Em posse de autorização dos órgáos oficiais promotores da competição, as maiores delegaçôes, com maior número de modalidades inscritas, foram procuradas sendo distribuídos 622 questionários aos atletas, sendo com a taxa de resposta de 72,7\%. Após entrega dos questionários, pesquisadores supervisionaram o preenchimento dos mesmos, os quais foram respondidos de forma anônima.

A pesquisa foi aprovada pelo Comitê de Ética e Pesquisa da Universidade do Estado de Santa Catarina (n. 666.548). Os atletas que aceitaram participar do estudo assinaram um Termo de Consentimento Livre Esclarecido e os atletas menores de 18 anos assinaram também o termo de Assentimento, bem como foi requerida a assinatura dos pais ou responsáveis, ou do técnico responsável quando necessário. A idade mínima para fazer parte da amostra foi de 14 anos.

Os atletas responderam a um questionário contendo informaçôes: dados pessoais (sexo e idade)

Foram analisadas as seguintes questôes relacionadas ao sono:

- duração do sono: analisada a partir dos horários de acordar e de dormir nos dias de semana ${ }^{17}$. A partir desta análise os atletas foram classificados como estáo recebendo alguma instrução sobre o sono na sua preparação. Assim, o objetivo desta pesquisa foi investigar algumas questóes de sono (duraçáo e qualidade do sono), saúde (percepção subjetiva de saúde) e treinamento (instrução sobre como deve ser seu sono durante o treinamento e/ou antes das competiçóes) de atletas de elite do Estado de Santa Catarina - SC.

tendo baixa duração do sono quando dormissem menos que oito horas.

- qualidade do sono: analisada por meio do Índice de Qualidade de Sono de Pittsburgh (PSQI) formado por questóes relacionadas ao sono de acordo com os seguintes componentes $\left.{ }^{18-19}: 1\right)$ qualidade subjetiva do sono; 2) latência do sono; 3) duração do sono; 4) eficiência habitual do sono; 5) distúrbios do sono; 6) uso de medicaçáo para dormir; 7) sonolência diurna e distúrbios durante o dia. Cada componente possui pontuaçóes especificas, sendo o máximo de 21 pontos. Escores superiores a cinco indicam qualidade ruim de sono.

- sono e pesadelos na véspera de competição: foram analisadas por meio das seguintes perguntas: "você costuma ter um sono pior na véspera de um jogo ou competiçáo importante comparado com uma noite normal?" (sim/não) e "você costuma ter pesadelos na véspera de um jogo ou competiçáo importante?" (sim/não) ${ }^{1}$.

Além disso, foi investigada a percepção subjetiva de saúde dos atletas por meio da seguinte pergunta: "Como você classifica seu estado de saúde atual?". Para esta questão foi considerada percepção positiva de saúde as respostas "excelente" e "boa" e percepção negativa de saúde as respostas "regular" e "ruim".

Por fim os atletas foram questionados se receberam instruçóes sobre sono durante seus treinamento por meio da seguinte questão: "durante sua preparação você recebeu instruçôes de como deve ser seu sono durante o treinamento e/ou antes das competições?” (sim; não).

Foram realizadas análises descritivas (médias, frequências e desvios padrôes). Para a comparaçáo das variáveis contínuas utilizou-se o teste de KruskalWallis e para comparação entre as proporçóes o teste do Qui-Quadrado completada pela análise do "Effect size". Com o objetivo de analisar as variáveis associadas à baixa duração de sono (menos de oito horas) e à baixa qualidade do sono utilizou-se a análise de regressão de Poisson ${ }^{20}$. Para o modelo ajustado considerou-se 
aqueles variáveis com $\mathrm{p}<0,25$ na análise não ajusta$\mathrm{da}^{21}$. Para todas as análises considerou-se um nível de probabilidade de confiança de 5\%.

\section{Resultados}

Os dados descritivos da amostra foram apresentados na TABELA 1 . Os atletas investigados apresentaram, em média, 21,16(5,83) anos com média de anos de prática da modalidade de nove anos e sete meses. Homens e mulheres apresentam duraçóes do sono semelhantes $(p=0,863)$. No entanto, as mulheres apresentaram maior prevalência de baixa qualidade do sono ( $\mathrm{p}=0,008)$.

A baixa duração do sono (menos que oito horas) foi identificada em boa parcela dos atletas (48,5\%) não sendo identificadas diferenças nesta variável entre as faixas etárias $(\mathrm{p}=0,427)$. A prevalência
Utilizou-se o "software" do programa estatístico The Statistical Package for the Social Sciences (SPSS) for Windows versão 20.0 geral de baixa qualidade do sono foi identificada em 38\% da amostra. Ainda considerando a baixa qualidade do sono identificou-se uma tendência dos atletas com mais de 18 anos apresentarem maior prevalência $(41,7 \%)$ do que os de 14 a 18 anos $(33,7 \%)(\mathrm{p}=0,098)$.

A maior parte dos atletas percebeu sua saúde como excelente ou boa $(89,6 \%)$ e recebeu instruções sobre sono durante seus treinamentos $(63,5 \%)$. Ainda, a maior parte dos atletas afirmou dormir pior que o habitual antes das competiçôes bem como a presença de pesadelos nestes períodos.

TABELA 1 - Características da amostra.

\begin{tabular}{|c|c|c|c|}
\hline \multirow{2}{*}{ Variáveis } & \multicolumn{2}{|c|}{ Sexo } & \multirow{2}{*}{ p valor } \\
\hline & Feminino $(n=276)$ & Masculino $(n=176)$ & \\
\hline Idade, anos & $20,31(5,30)$ & $22,48(6,36)$ & $<0,001$ \\
\hline \multicolumn{4}{|l|}{ Faixa etária, (anos)\% } \\
\hline $14-18$ & $53,9(\mathrm{n}=65)$ & $43,0(\mathrm{n}=37)$ & 0,034 \\
\hline Mais de 18 & $46,1(n=86)$ & $57,0(\mathrm{n}=117)$ & \\
\hline Duração do sono, $\mathrm{h}$ & $7,92(1,36)$ & $7,88(1,60)$ & 0,863 \\
\hline Baixa duração do sono, $(<8 \mathrm{~h}) \%$ & $49,3(\mathrm{n}=134)$ & $47,3(\mathrm{n}=79)$ & 0,690 \\
\hline Baixa qualidade do sono, $\%$ & $42,9(\mathrm{n}=117)$ & $30,2(\mathrm{n}=52)$ & 0,008 \\
\hline \multicolumn{4}{|l|}{ Percepção de saúde, \% } \\
\hline Excelente & $32,1(\mathrm{n}=87)$ & $42,2(n=73)$ & 0,080 \\
\hline Boa & $57,9(\mathrm{n}=157)$ & $50,9(\mathrm{n}=88)$ & \\
\hline Regular/ruim & $10,0(\mathrm{n}=27)$ & $6,9(\mathrm{n}=12)$ & \\
\hline \multicolumn{4}{|c|}{ Instrução do sono durante o treinamento, \% } \\
\hline Sim & $63,5(n=174)$ & $63,2(\mathrm{n}=109)$ & 0,951 \\
\hline Não & $36,5(\mathrm{n}=99)$ & $36,8(n=64)$ & \\
\hline \multicolumn{4}{|c|}{ Pesadelo(s) durante a noite antes de uma competição importante, \% } \\
\hline Sim & $73,5(\mathrm{n}=197)$ & $83,1(n=143)$ & 0,019 \\
\hline Não & $26,5(n=71)$ & $16,9(\mathrm{n}=29)$ & \\
\hline \multicolumn{4}{|c|}{ Dormir pior durante a noite (s) antes de uma competição importante ou jogo, \% } \\
\hline Sim & $65,0(\mathrm{n}=178)$ & $57,2(\mathrm{n}=99)$ & 0,101 \\
\hline Não & $35,0(\mathrm{n}=96)$ & $42,8(\mathrm{n}=74)$ & \\
\hline
\end{tabular}


Identificou-se que aqueles atletas que receberam instruçóes sobre sono nos seus treinamentos apresentaram maior duraçáo do sono $(\mathrm{p}<0,001)$ e menor PSQI $(\mathrm{p}=0,045)$ indicando melhor qualidade do sono (FIGURAS 1 e 2). Com relação à duração do sono este resultado foi semelhante quando estratificado por sexo sendo a duração do sono superior, tanto nos homens ( $p=0,001)$, como nas mulheres $(p=0,008)$. Já considerando o PSQI a tendência dos atletas que tiveram instruçôes sobre o sono apresentarem melhores indicadores de qualidade do sono foi identificada apenas nas mulheres ( $\mathrm{p}=0,008)$, não sendo observada diferença no sexo masculino $(\mathrm{p}=0,946)$. Além disso, aqueles atletas que receberam instruçóes sobre o sono durante os treinamentos apresentaram menor frequência de pesadelos $(\mathrm{p}=0,001)$ na véspera de competiçôes importantes, tendência observada para ambos os sexos. Quando esta análise foi estratificada pelas faixas etárias esta associação foi mais evidente naqueles atletas maiores de 18 anos $(\mathrm{p}=0,030)$.

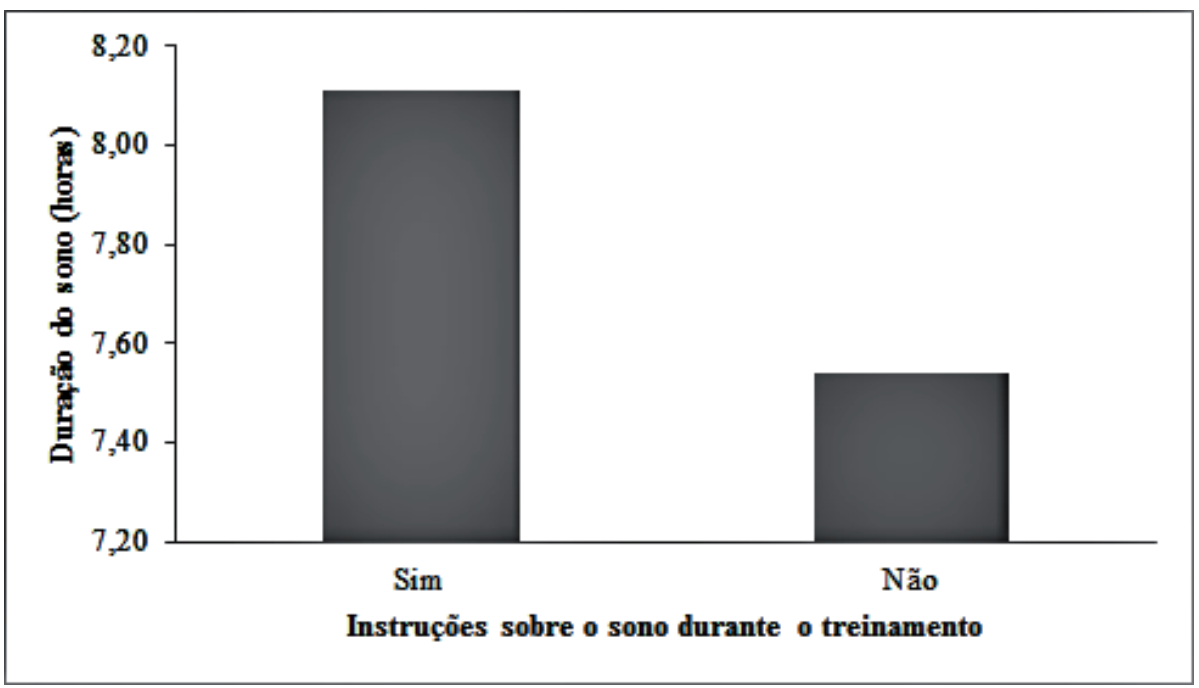

FIGURA 1 - Associação entre a duração do sono e as instruções do sono durante o treinamento.

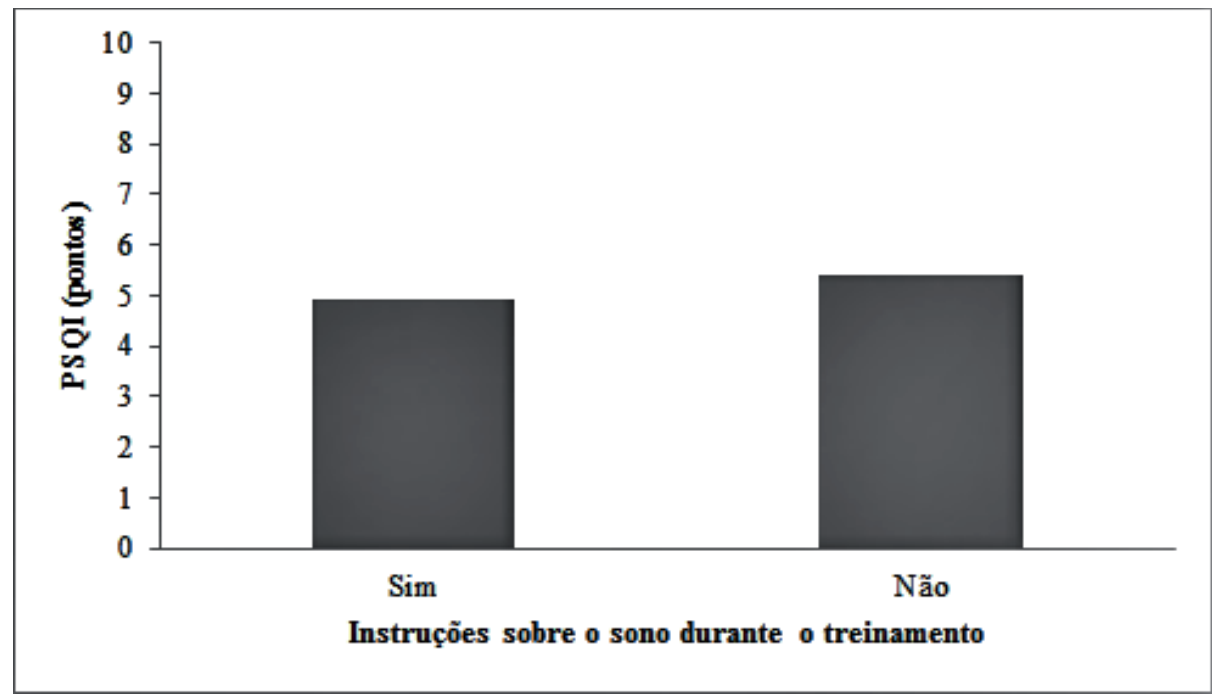

FIGURA 2 - Associação entre pontuação do PSQI e as instruções do sono durante o treinamento. 
Nas TABELAS 2 e 3 foram apresentadas análises de associação considerando a baixa duração e a baixa qualidade do sono como variáveis dependentes. Considerando a baixa duração do sono foram identificadas associações nas análises bivariadas com as variáveis qualidade do sono $(\mathrm{p}<0,001)$ e instruçōes sobre o sono durante os treinamentos ( $\mathrm{p}=0,001)$. Estas variáveis se mantiveram associadas com a variável dependente na análise ajustada sendo que os atletas que não tiveram instruçôes sobre o sono durante seus treinamentos apresentaram 1,35 (IC95\%1,02-1,78) vez mais prevalência de baixa duração do sono que aqueles que receberam.

TABELA 2 - Prevalências e razões de prevalências (RP) considerando a baixa duração do sono como variável dependente.

\begin{tabular}{|c|c|c|c|c|c|}
\hline \multirow[b]{2}{*}{ Variáveis } & \multirow[b]{2}{*}{$\begin{array}{c}\text { Prevalências } \\
(\%)\end{array}$} & \multicolumn{2}{|c|}{ Análise bruta* } & \multicolumn{2}{|c|}{ Análise ajustada** } \\
\hline & & $\begin{array}{c}\text { RP } \\
(95 \% \text { IC) }\end{array}$ & p-valor & $\begin{array}{c}\text { PR } \\
(95 \% \text { IC) }\end{array}$ & p-valor \\
\hline \multicolumn{6}{|l|}{ Idade, anos } \\
\hline $14-18$ & 45,2 & 1 & 0,426 & Excluído & - \\
\hline Mais de 18 & 49,2 & $1,09(0,89-1,34)$ & & & \\
\hline \multicolumn{6}{|l|}{ Sexo } \\
\hline Masculino & 52,7 & $1,04(0,86-1,25)$ & 0,690 & Excluído & - \\
\hline Feminino & 50,7 & 1 & & & \\
\hline \multicolumn{6}{|l|}{ Qualidade do sono } \\
\hline Boa & 38,6 & 1 & $<0,001$ & 1 & 0,001 \\
\hline Ruim & 63,7 & $1,65(1,37-2,00)$ & & $1,59(1,21-2,09)$ & \\
\hline \multicolumn{6}{|l|}{ Percepção de saúde } \\
\hline Positiva & 47,2 & 1 & 0,208 & 1 & 0,696 \\
\hline Negativa & 57,9 & $1,23(0,92-1,64)$ & & $1,09(0,70-1,71)$ & \\
\hline \multicolumn{6}{|c|}{ Instrução sobre o sono durante o treinamento } \\
\hline Sim & 42,4 & 1 & 0,001 & 1 & 0,033 \\
\hline Não & 59,5 & $1,41(1,17-1,71)$ & & $1,35(1,02-1,78)$ & \\
\hline \multicolumn{6}{|c|}{ Pesadelo antes de competição/jogo importante } \\
\hline Sim & 49,1 & $1,12(0,87-1,44)$ & 0,363 & Excluído & - \\
\hline Não & 43,9 & 1 & & & \\
\hline \multicolumn{6}{|c|}{ Dormir pior antes de competição ou jogo importante } \\
\hline Sim & 46,3 & 1 & 0,290 & Excluído & - \\
\hline Não & 51,5 & $1,11(0,92-1,35)$ & & & \\
\hline
\end{tabular}

Considerando a baixa qualidade do sono como variável dependente foram identificadas associações na análise não ajustadas com as variáveis, sexo $(\mathrm{p}=$ $0,008)$, duração do sono $(\mathrm{p}<0,001)$, percepção de saúde $(\mathrm{p}=0,004)$ e receber instruçóes sobre o sono durante os treinamentos $(\mathrm{p}=0,044)$. $\mathrm{Na}$ análise ajustada permaneceram associadas às variáveis, sexo, duração do sono e percepçáo de saúde sendo que aqueles atletas com percepçáo negativa de saúde apresentação 1,58 (IC95\% 1,01-2,48) vez mais prevalência de baixa qualidade do sono que aqueles que receberam instruçōes. *análise bruta utilizando o p-valor do teste do Qui-quadrado.

**análise ajustada considerando as variáveis com $p<0,25$ na análise bruta. 
*análise bruta utilizando o p-valor do teste do Qui-quadrado.

**análise ajustada considerando as variáveis com com $p<0,25$ na análise bruta.
TABELA 3 - Prevalências e razões de prevalências (RP) considerando a baixa qualidade do sono como variável dependente.

\begin{tabular}{|c|c|c|c|c|c|}
\hline \multirow[b]{2}{*}{ Variáveis } & \multirow[b]{2}{*}{$\begin{array}{c}\text { Prevalências } \\
(\%)\end{array}$} & \multicolumn{2}{|c|}{ Análise bruta* } & \multicolumn{2}{|c|}{ Análise ajustada** } \\
\hline & & $\begin{array}{c}\text { RP } \\
(95 \% \text { IC) }\end{array}$ & p-valor & $\begin{array}{c}\text { PR } \\
(95 \% \text { IC) }\end{array}$ & p-valor \\
\hline \multicolumn{6}{|l|}{ Idade, anos } \\
\hline $14-18$ & 33,7 & 1 & 0,098 & 1 & 0,122 \\
\hline Mais de 18 & 41,7 & $1,23(0,96-1,60)$ & & $1,29(0,93-1,80)$ & \\
\hline \multicolumn{6}{|l|}{ Sexo } \\
\hline Masculino & 30,2 & 1 & 0,008 & 1 & 0,023 \\
\hline Feminino & 42,9 & $1,42(1,08-1,84)$ & & $1,54(1,06-2,24)$ & \\
\hline \multicolumn{6}{|l|}{ Duração do sono } \\
\hline$<8$ horas & 51,0 & $1,88(1,46-2,42)$ & $<0,001$ & 1 & $<0,001$ \\
\hline 8 horas ou mais & 27,1 & 1 & & $1,87(1,32-2,64)$ & \\
\hline \multicolumn{6}{|l|}{ Percepção de saúde } \\
\hline Positiva & 35,7 & 1 & 0,004 & 1 & 0,044 \\
\hline Negativa & 59,0 & $1,65(1,23-2,21)$ & & $1,58(1,01-2,48)$ & \\
\hline \multicolumn{6}{|c|}{ Instrução sobre o sono durante o treinamento } \\
\hline Sim & 34,4 & 1 & 0,044 & 1 & 0,410 \\
\hline Não & 44,1 & $1,28(1,01-1,63)$ & & $1,15(0,82-1,61)$ & \\
\hline \multicolumn{6}{|c|}{ Pesadelo antes de competição/jogo importante } \\
\hline Sim & 37,0 & $1,02(0,80-1,40)$ & 0,713 & Excluído & - \\
\hline Não & 36,0 & 1 & & & \\
\hline \multicolumn{6}{|c|}{ Dormir pior antes de competição / jogo importante } \\
\hline Sim & 37,3 & $1,03(0,82-1,33)$ & 0,714 & Excluído & - \\
\hline Não & 36,1 & 1 & & & \\
\hline
\end{tabular}

\section{Discussão}

Este estudo buscou investigar, em atletas de elite do Estado de Santa Catarina - SC, algumas questóes de sono, saúde e treinamento. Destaca-se que, por se tratar de amostra representativa de atletas de Santa Catarina e por ser um estudo exploratório, visto as limitadas informaçóes sobre sono de atletas brasileiros, tais resultados podem servir para se traçar um perfil destes atletas, bem como propor estratégias, visando, tanto a promoção da saúde, como a melhoria do desempenho atlético. Aponta-se como limitações a utilização de questionários para identificação das variáveis relacionadas ao sono, a limitação geográfica da coleta de dados e a não descrição das instruçôes sobre sono fornecidas pelos treinadores.

Identificou-se que mais de $70 \%$ dos atletas apresentavam pesadelos antes de jogos e competiçóes importantes, bem como, elevado percentual percebiam seu sono pior nestes períodos, o que remete à necessidade de maior observação destas questóes considerando o desempenho destes atletas. Este resultado é semelhante ao observado por ERLACHER et al. ${ }^{1}$ em atletas da elite olímpica alemã, na qual a maioria relatou dormir pior durante a noite anterior às competiçóes bem como apresentava pesadelos, especialmente considerando as mulheres. Além de questôes relacionadas aos pensamentos sobre a competição e nervosismo ERLACHER et al. ${ }^{1}$ destacam que ruídos nos alojamentos, o nível das competiçôes e a cobrança por resultados podem exacerbar problemas com sono durante estes períodos.

A baixa duração do sono (menos que oito horas) foi identificada em boa parcela dos atletas $(48,5 \%)$ não sendo identificadas diferenças nesta variável entre as faixas etárias $(p=0,427)$. A literatura é restrita considerando possíveis pontos de corte para baixa duração 
do sono para a população em geral e, em especial, para atletas. O estudo de Pereira et al..$^{22}$, com adolescentes brasileiros, comparou as prevalências de baixa duração do sono entre trabalhadores e não trabalhadores. A prevalência de baixa duração do sono nos atletas analisados foi semelhante à observada no grupo de trabalhadores (52\%) e superior àquela observada no grupo de não trabalhadores $(36,2 \%)$ o que poderia indicar que o treinamento esportivo interfere nas rotinas diárias e de sono dos atletas de forma semelhante ao que acontece com as rotinas de trabalho.

Considerando a duraçáo média do sono nos atletas de Santa Catarina (7,90 horas) é possível identificar diferenças considerando estudos publicados com atletas estrangeiros. No estudo de BrAND et al. ${ }^{4}$, com atletas adolescentes, foi observada uma duração média de 8,60 horas de sono. Já o estudo de LeEDER et al. ${ }^{23}$ em atletas de nível olímpico foi observada uma média de sono de 6,91 horas. Em atletas universitários МaH et al. ${ }^{2}$ observaram uma média de duração de sono de 7,83 horas. Assim, parece que a duração do sono no atleta poderia estar ligada ao seu nível de desempenho, sendo identificada menor duração nos atletas de elite em relação aos demais. Apesar disso, é preciso estudos que forneçam mais dados que indiquem possíveis recomendaçóes de duração do sono para atletas, o efeito destas duraçóes no desempenho atlético, bem como a organização do volume, intensidade e horários dos treinamentos.

Percebe-se que receber instruçôes sobre o sono está associado com duração e qualidades do sono mais adequadas, entretanto, neste estudo, não foi verificado quais as instruçōes que foram dadas e como e qual momento do treinamento elas chegaram ao atleta.

$\mathrm{Na}$ análise de associação, na qual se considerou a baixa duraçáo do sono como variável dependente, identificaram-se associações, na análise ajustada, da variável dependente com a baixa qualidade de sono (p $<0,001)$ e com a variável "receber instruçóes sobre o sono durante o treinamento" $(\mathrm{p}=0,001)$. Especificamente em relação à associação com instrução sobre o sono, percebe-se que, de fato, é importante orientar os atletas em relação às estratégias e rotinas, que podem se constituir em mudanças de hábitos que permitam um sono eficiente e com duração adequada.

A literatura é restrita considerando tais recomendaçôes bem como estratégias práticas para melhorar o sono dos atletas. Apesar disso, a partir do estudo de Fietze et al. ${ }^{12}$, com sono de bailarinos do Ballet Nacional de Berlim, foi criada uma sala de cochilos visando a recuperaçáo do déficits de sono. Assim, além de instruçôes, faz-se necessário a criação de um conjunto de estratégias que permita que os atletas tenham possibilidades reais de adequar suas necessidades de sono às demandas e horários de treinamentos.

Neste contexto МAH et al. ${ }^{2}$ investigaram o efeito da extensão do sono (dormir mais de que de costume) no rendimento de atletas de basquete. Neste estudo os atletas receberam a instruçáo de dormir duas horas a mais que seu habitual. Como resultado verificou-se rendimento superior em algumas medidas de desempenho como a precisão no arremesso da bola ao cesto o que sugere que os atletas estavam tendo uma duração de sono aquém de suas necessidades. Além disso, Azboy e KaYGisiz ${ }^{11}$ investigando atletas de voleibol e atletismo, verificaram que a privação do sono diminuiu a eficiência cognitiva e a motivaçáo para o treinamento gerando aumento no cansaço, diminuiçáo da motivação e da atenção e pior desempenho em teste de tempo de reação.

Já as prevalências de baixa qualidade do sono nos atletas de SC (42,9\% para as mulheres e 30,2\% para os homens) foi inferior à observada no estudo de Pereira et al. ${ }^{24} \mathrm{em}$ músicos de orquestras $(71,4 \%)$ e no estudo de Martini et al. ${ }^{25}$ com estudantes universitários $(51,75 \%)$. Além disso, duração do sono e qualidade do sono não são, necessariamente, variáveis associadas. As associaçóes entre baixa duração de sono e pior qualidade de sono observadas nos atletas também foram identificadas no estudo de MARTINI et al..$^{25}$. Assim, considerando que, em tese, os atletas possuam uma melhor qualidade do sono comparado aos grupos acima citados é possível discutir a hipótese que o treinamento físico possa influenciar na expressão do seu ciclo vigília/sono, modificando, por exemplo, o nadir da temperatura corporal ${ }^{26}$.

Investigando a associação entre a variável qualidade de sono e percepção de saúde nos atletas de $\mathrm{SC}$, observou-se que os atletas com pior qualidade de sono tiveram uma percepção de saúde mais negativa $(\mathrm{p}=0,030)$. Este resultado corrobora os achados de FonseCA et al. ${ }^{27}$ que identificaram que trabalhadores relataram que a prevalência de percepção negativa de saúde foi significativamente superior naqueles sujeitos com percepção negativa de sono. Também com um grupo de trabalhadores, neste caso em músicos de orquestras, Pereira et al. ${ }^{24}$ identificaram que uma melhor qualidade de sono apresentou associação significativa com melhor qualidade de vida bem como percepção de geral saúde.

Especificamente com atletas a literatura é restrita ao descrever tais relaçôes, mas já foram identificadas em estudo com dançarinos profissionais ${ }^{12}$, associações significativas entre qualidade do sono e 
qualidade de vida relacionada à saúde. Além disso, na população em geral, conforme observado por Geiger et al. ${ }^{28}$, uma percepçáo negativa de saúde está associada com a percepção de sono insuficiente. Entende-se, desta forma, que um sono de qualidade seja necessário, não somente com vistas ao desempenho atlético, mas para a preservação da saúde geral do atleta que, muitas vezes, precisa conviver com questóes que podem piorar sua qualidade de vida como lesôes, pressão por resultados e condições ruins de trabalho e treinamento. Além disso, outro fator importante a ser investigados em futuros estudos com o sono do atleta é a ansiedade, especialmente na pré-competição.

Importante destacar nesta discussão que o exercício físico seja ele competitivo ou para a saúde, pode modificar a estrutura do sono bem como sua eficiência e latência e que o tipo e horários de treinamento precisam ser considerados nesta discussão como constatado nos estudos de Williams et al. ${ }^{29}$, Driver et al..$^{30}$ e Honer e Porter ${ }^{31}$. Neste contexto em seu estudo de revisão sobre o sono e exercício MARTINS et al. ${ }^{32}$ discutem que conhecer melhor o padrão do sono de quem pratica exercício físico é necessário pois tal associação pode ser determinando para a melhoria do desempenho motor bem como proteção de sintomas de estresse e ansiedade durante os treinamentos.

De forma geral os resultados das análises de sono dos atletas de elite do Estado de Santa Catarina remetem a altas prevalências de baixa qualidade do sono nos atletas investigados. Além disso, a maioria dos atletas relatou que seu sono piora na véspera de competiçôes e jogos importante. Uma boa qualidade do sono esteve associada com percepçáo mais positiva de saúde e aqueles atletas que receberam instruçóes sobre o sono durante seus treinamentos apresentaram maior duração e melhor qualidade do sono. Recomenda-se, desta forma, que as instruçóes sobre sono passem a fazer parte das rotinas de treinamentos de atletas brasileiros, bem como se investigue quais informaçóes e estratégias são mais efetivas para melhora do sono dos atletas.

\begin{abstract}
Sleep in elite athletes in the State of Santa Catarina, Brazil.

This study aimed to investigate some questions about sleep, health and training of elite athletes from the State of Santa Catarina. A sample was constituted of 452 athletes of collective and individual sports who responded to a questionnaire with questions about sleep (duration and quality, nightmares and worsening of sleep before the competition) on their health perception. Furthermore, it was investigated if the athletes received, during their trainings, recommendations regarding their sleep. $48.5 \%$ of athletes presented low sleep duration $(<8 \mathrm{~h})$. Those who received instructions about the sleep in training presented higher sleep duration $(p<0.001)$ and higher sleep quality $(p=0.045)$. Athletes that didn't have instructions about sleep presented 1.35 times (Cl95\% 1.02-1.78) more prevalence of low sleep duration. Furthermore, most athletes reported that their sleep gets worse on the eve of competitions and important games. A good sleep quality was associated with positive health perception, and those athletes who received instructions about their sleep during their trainings presented higher duration and better sleep quality. It is recommended that instructions about sleep become part of training routines of Brazilian athletes, well as investigate which information and strategies are more effective to improve the sleep of athletes.
\end{abstract}

KEY WoRDS: Sleep deprivation; Athletes; Health.

\title{
Referências
}

1. Erlacher D, Ehrlenspiel F, Adegbesan OA, El-Din HG. Sleep habits in German athletes before important competitions or games. J Sports Sci. 2011;29:271-82.

2. Mah CD, Mah KE, Kezirian EJ, Dement WC. The effects of sleep extension on the athletic performance of collegiate basketball players. Sleep. 2011;34:943-50. 
3. Belenky G, Wesensten NJ, Thorne DR, et al. Patterns of performance degradation and restoration during sleep restriction and subsequent recovery: a sleep dose-response study. J Sleep Res. 2003;12:1-12.

4. Brand S, Beck J, Gerber M, Hatzinger M, Holsboer-Trachsler E. Football is good for your sleep: favorable sleep patterns and psychological functioning of adolescent male intense football players compared to controls. J Health Psychol. 2009; 14:1144-55.

5. Gerber M, Holsboer-Trachsler E, Pühse U, Brand S.Elite sport is not an additional source of distress for adolescents with high stress levels. Percept Mot Skills. 2011;112:581-99.

6. Walker MP, Brakefield T, Morgan A, Hobson JA, Stickgold, R. Practice with sleep makes perfect: sleep-dependent motor skill learning. Neuron. 2002;35:205-11.

7. Postolache TT. Sports chronobiology. Clin Sports Med. 2005;24:xix-xxii.

8. Leger D, Elbaz M, Raffray T, Metlaine A, Bayon V, Duforez F. Sleep management and the performance of eight sailors in the Tour de France a la voile yacht race. J Sports Sci. 2008;2:21-8.

9. Mah CD, Mah KE, Dement WC. Athletic performance improvements and sleep extension in collegiate tennis players. Sleep. 2009;32:A155.

10. Erlacher D, Schredl M, Ehrlenspiel F, Bosing M. Subjective sleep quality and state anxiety of high-school students prior to a final sport exam. Adv Psychol Res. 2009; 63:179-86.

11. Azboy O, Kaygisiz Z. Effects of sleep deprivation on cardiorespiratory functions ofthe runners and volleyball players during rest andexercise. Acta Physiol Hung. 2009;96:29-36.

12. Fietze I, Strauch J, Holzhausen M, et al. Sleep quality in professional ballet dancers. Chronobiol Int. 2009; 26:1249-62.

13. Haddad M, Chaouachi A, Wong P, et al. Influence of fatigue, stress, muscle soreness and sleep on perceived exertion during submaximal effort. Physiol Behav. 2013;2:185-9.

14. Kennedy MD, Tamminen KA.. Factors that influence fatigue status in Canadian university swimmers. J Sports Sci. 2012;31:554-64.

15. Mihalik JP, Lengas E, Register-Mihalik JK, Oyama S, Begalle RL, Guskiewicz KM. The effects of sleep quality and sleep quantity on concussion baseline assessment. Clin J Sport Med. 2013;23:343-8.

16. Rodrigues PC. Bioestatística. 3a ed. Niterói: Editora da Universidade Federal Fluminense; 2002.

17. Louzada FM, Menna-Barreto L. Sleep-wake cycle in rural populations. Biol Rhythm Res. 1986;35:153-7.

18. Buysse DJ, Reynolds CF, 3rd Monk TH, Berman SR, Kupfer DJ. The Pittsburgh Sleep Quality Index: a new instrument for psychiatric practice and research. Psychiatry Res. 1989;28:193-213.

19. Bertolazi AN, Fagondes SC, Hoff LS, et al. Validation of the Brazilian Portuguese version of the Pittsburgh Sleep Quality Index. Sleep Med. 2011;12:70-5.

20. Barros AJ, Hirakata VN. Alternatives for logistic regression in cross-sectional studies: an empirical comparison of models that directly estimate the prevalence ratio. BMC Med Res Methodol. 2003;3:21.

21. Hosmer DW, Leneshow S. Applied logistic regression. New York: Wiley; 1989.

22. Pereira EF, Bernardo MPSL, D’Almeida V, Louzada FM. Sono, trabalho e estudo: duraçáo do sono em estudantes trabalhadores e não trabalhadores. Cad Saúde Públ. 2011; 27:975-84.

23. Leeder J, Glaister M, Pizzoferro K, Dawson J, Pedlar C. Sleep duration and quality in elite athletes measured using wristwatch actigraphy. J Sports Sci. 2012;30:541-45.

24. Pereira ÉF, Teixeira CS, Kothe F, Merino EAD, Daronco LSE. Percepção de qualidade do sono e da qualidade de vida de músicos de orquestra. Rev Psiq Clín. 2010;37:48-51.

25. Martini M, Brandalize M, Louzada FM, Pereira ÉF, Brandalize D. Fatores associados à qualidade do sono em estudantes de Fisioterapia. Fisioter Pesq. 2012;19:261-67.

26. Van Reeth O, Sturis J, Byrne MM, et al. Nocturnal exercise phase delays circadian rhythms of melatonin and thyrotropin secretion in normal men. Am J Physicol. 1994; 266:E964-E74.

27. Fonseca SA, Blank VLG, Barros MVG, Nahas MV. Percepçáo de saúde e fatores associados em industriários de Santa Catarina, Brasil. Cad Saúde Públ. 2008;24:567-76.

28. Geiger SD, Sabanayagam C, Shankar A. The relationship between insufficient sleep and self-rated health in a nationally representative sample. J Environ Public Health. 2012;2012:518263.

29. Williams HL, Hammack JT, Daly RL, Dement WC, Lubin A. Responses to auditory stimulation, sleep loss and the EEG stages of sleep. Electro-encephalogr Clin Neurophysiol. 1964;16:269-79.

30. Driver HS, Rogers GG, Mitchell D, et al. Prolonged endurance exercise and sleep disruption. Med Sci Sports Exerc 1994;26:903-7. 
Bleyer FTS, et al.

31. Horne JA, Porter JM. Exercise and human sleep. Nature 1975;256:573-5.

32. Martins PJF, Mello MT, Tufik S. Exercicio e sono. Rev Bras Med Esporte. 2001;7:1.

\begin{tabular}{r|l} 
ENDEREço & \\
Fernanda Tolentino de Souza Bleyer & \\
Centro de Ciências da Saúde e do Esporte & Recebido para publicação: 29/07/2014 \\
Universidade do Estado de Santa Catarina & 1a. Revisão: 21/12/2015 \\
R. Pascoal Simone, 358 & 2a. Revisão: 27/02/2015 \\
88080-350 - Florianópolis - SC - BRASIL & Aceito: 19/05/2015 \\
e-mail: fernanda-ts@uol.com.br & \\
&
\end{tabular}

216 • Rev Bras Educ Fís Esporte, (São Paulo) 2015 Abr-Jun; 29(2):207-16 